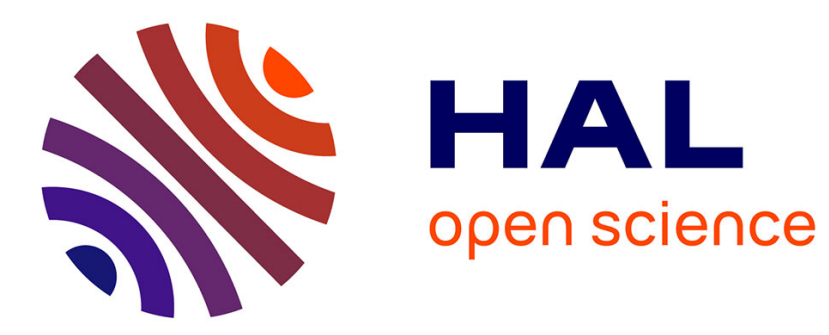

\title{
Southern Hemisphere Jet Variability in the IPSL GCM at Varying Resolutions
}

Ara Arakelian, Francis Codron

\section{To cite this version:}

Ara Arakelian, Francis Codron. Southern Hemisphere Jet Variability in the IPSL GCM at Varying Resolutions. Journal of the Atmospheric Sciences, 2012, 69 (12), pp.3788-3799. 10.1175/jas-d-120119.1. hal-01115487

\author{
HAL Id: hal-01115487 \\ https://hal.science/hal-01115487
}

Submitted on 11 Feb 2015

HAL is a multi-disciplinary open access archive for the deposit and dissemination of scientific research documents, whether they are published or not. The documents may come from teaching and research institutions in France or abroad, or from public or private research centers.
L'archive ouverte pluridisciplinaire HAL, est destinée au dépôt et à la diffusion de documents scientifiques de niveau recherche, publiés ou non, émanant des établissements d'enseignement et de recherche français ou étrangers, des laboratoires publics ou privés. 


\title{
Southern Hemisphere Jet Variability in the IPSL GCM at Varying Resolutions
}

\author{
ARA ARAKELIAN AND FRANCIS CODRON \\ Laboratoire de Météorologie Dynamique, Université Pierre et Marie Curie, CNRS, Paris, France
}

(Manuscript received 16 April 2012, in final form 11 July 2012)

\begin{abstract}
Fluctuations of the Southern Hemisphere eddy-driven jet are studied in a suite of experiments with the Laboratoire de Météorologie Dynamique, version 4 (LMDZ4) atmospheric GCM with varying horizontal resolution, in coupled mode and with imposed SSTs. The focus is on the relationship between changes in the mean state brought by increasing resolution, and the intraseasonal variability and response to increasing $\mathrm{CO}_{2}$ concentration.

In summer, the mean jet latitude moves poleward when the resolution increases in latitude, converging toward the observed one. Most measures of the jet dynamics, such as skewness of the distribution or persistence time scale of jet movements, exhibit a simple dependence on the mean jet latitude and also converge to the observed values. In winter, the improvement of the mean-state biases with resolution is more limited.

In both seasons, the relationship between the dominant mode of variability - the southern annular mode (SAM) - and the mean state remains the same as in observations, except in the most biased winter simulation. The jet fluctuations-latitude shifts or splitting_-just occur around a different mean position. Both the model biases and the response to increasing $\mathrm{CO}_{2}$ project strongly onto the SAM structure. No systematic relation between the amplitude of the response and characteristics of the control simulation was found, possibly due to changing dynamics or impacts of the physical parameterizations with different resolutions.
\end{abstract}

\section{Introduction}

The atmospheric variability over the Southern Ocean is dominated at time scales longer than a week by zonally symmetric meridional fluctuations of the jet, a structure known as the southern annular mode (SAM). The SAM also has a large impact on the ocean (Sen Gupta and England 2006) and often dominates the regional response to external forcings, such as greenhouse gas increase, ozone depletion (Gillett and Thompson 2003; Perlwitz et al. 2008; Son et al. 2010), or El Niño (L'Heureux and Thompson 2006).

This prominence of the SAM is thought to be partly due to a positive feedback with the momentum transport by eddies. In a zonally averaged, vertically integrated picture, changes in the convergence of eddy momentum fluxes force the fluctuations of the jet, which are then damped by surface friction or other mechanisms. Following the notations from Lorenz and Hartmann (2001),

Corresponding author address: A. Arakelian, Laboratoire de Météorologie Dynamique, Boite 99, 4 Place Jussieu, F-75252 Paris, France.

E-mail: aalmd@lmd.jussieu.fr the evolution of an index $z(t)$ of the zonal-mean jet variability, such as the SAM, can be written as

$$
\partial_{t} z=m-z / \tau
$$

where $\tau$ is a damping time scale approximating the impacts of surface friction and other processes, and $m$ is an index of the forcing of $z$ by eddy momentum fluxes. If the eddies are partially organized by changes in the mean flow, then a positive feedback will result if they respond in a way that reinforces the mean-flow changes. In its simplest form, the eddy forcing can then be decomposed into

$$
m=\tilde{m}+b z
$$

where $b$ is a feedback coefficient and $\tilde{m}$ is the fraction of $m$ that is independent of $z$, usually modeled as a random process. The presence of $b$ will increase the variance of $z$ at low frequencies and also amplify the response to an external forcing: adding a stationary forcing $F$ to the right-hand side of Eq. (1), the stationary response becomes 


$$
\bar{z}=F \times \frac{\tau}{1-b \tau} .
$$

If the damping time scale is the same for all modes of variability, then the modes with a stronger feedback will be prominent in the response, even if the initial forcing projects onto several modes.

Most atmospheric general circulation models share the same biases in their representation of the mean state and variability over the Southern Ocean: the mean jet is located too far equatorward, and the SAM is too persistent. Both seem related: models from the phase 3 of the Coupled Model Intercomparison Project (CMIP3) database that have the most equatorward jet also tend to have the most persistent SAM-or strongest eddy feedback (Barnes and Hartmann 2010b; Kidston and Gerber 2010). Consistently with the relation (3), they also have the strongest response to an external forcing, such as a $\mathrm{CO}_{2}$ increase. This relation between the jet latitude and the SAM persistence was also observed in dynamical core models (Gerber and Vallis 2007) when varying different parameters, but its cause is still debated. Barnes et al. (2010) proposed an explanation involving changes in the meridional propagation and breaking of waves at upper levels. An alternative theory (Robinson 2006) focuses on the location of near-surface eddy source regions, which could be more influenced by a more baroclinic jet (Chen and Plumb 2009). In full GCMs at least, both the equatorward position and the strong feedback could also be caused by a third unknown model bias, affecting, for example, the wave dynamics or the diabatic heating.

While many of the previously quoted studies of the SAM analyze the whole year, the SAM in fact exhibits a seasonal variability, both in its structure and in the observed eddy feedback (Codron 2007; Watterson 2007; Barnes and Hartmann 2010a). In summer, the climatology is more zonally uniform, and so is the SAM structure, which represents meridional wandering of the zonal-mean jet. Observed feedbacks are strongest in this season. In winter, departures from zonal symmetry are stronger; in particular over the Pacific Ocean, the SAMrelated variability is a seesaw between two distinct positions of the jet, instead of a meridional meandering. The eddy feedback is also weaker and confined to a restricted range of longitudes.

This paper analyses the variability of the Southern Hemisphere jet in a series of simulations with the Laboratoire de Météorologie Dynamique, version 4 (LMDZ4) atmospheric general circulation model with varying horizontal resolution, both in coupled and atmosphere-only configurations. The objectives are twofold:
- Assess how much the model biases in the representation of the mean state and variability improve with increased resolution.

- Use the resulting mean-state changes to study the relations between the mean state, the variability, and the response to increased $\mathrm{CO}_{2}$.

This study complements previous ones that used multimodel databases: there is less model variety, but the changes between simulations are better controlled. The range of mean states covered, as measured by the mean jet latitude, is similar in both cases. We also check in a more detailed way the seasonality and the zonal structure of the circulation changes.

The paper is organized as follows: section 2 presents the simulations that will be analyzed and section 3 the analysis methods. The results are then presented in section 4 for the summer season and section 5 for winter.

\section{Model description}

The simulations used in the paper are described in greater detail in Hourdin et al. (2012). They all use an identical version of the LMDZ4 atmospheric general circulation model, with 19 levels on the vertical but with varying horizontal resolution. LMDZ4 was the atmospheric component of the L'Institut Pierre-Simon Laplace Coupled Model, version 4 (IPSL CM4) (Marti et al. 2010), that participated in the CMIP3 experiment, coupled with the Organizing Carbon and Hydrology in Dynamic Ecosystems (ORCHIDEE) land surface model (Krinner et al. 2005) and the Nucleus for European Modelling of the Ocean (NEMO) oceanic GCM in the global ORCA2 configuration. The physical parameterizations are described in Hourdin et al. (2006); they are identical in all the simulations and there was no retuning of parameters. The parameterizations are not, however, "scale aware," and could behave somewhat differently at different resolutions; for example, precipitation tends to be more spatially concentrated at higher resolutions.

The dynamical part of the code is based on a finitedifference formulation of the primitive equations on a longitude-latitude Arakawa C-grid. The lowest resolution uses 96 points in longitude by 71 points in latitude, yielding a resolution of $3.75^{\circ} \times 2.5^{\circ}$. This resolution was used in the IPSL CM4 coupled GCM for the CMIP3 experiment. The number of points is then increased alternatively in latitude and in longitude, up to a doubling of the initial resolution. The complete set of configurations used is $96 \times 71,96 \times 95,144 \times 95,144 \times 142$, and $192 \times 142$. In addition to the resolution itself, the only changes between the different simulations are the 
decrease in the dynamical time step with increasing resolution and a decrease of the horizontal dissipation time scale for the two highest resolutions. Both changes were shown in experiments with the LMDZ dynamical core to have a much smaller impact than the resolution itself (Guemas and Codron 2011).

In the first series of experiments, the observed Atmospheric Model Intercomparison Project (AMIP) SSTs (Hurrell et al. 2008) from 1950 to 2007 are prescribed as a boundary condition over the oceans. These simulations will be identified by the prefix LMDZ4. In a second series, the LMDZ4 model is coupled to the NEMO ocean GCM. The same set of horizontal resolutions as in the first series is used in the atmosphere, but the ocean model does not vary: the only change is a slightly lower oceanic albedo at the lowest resolution to compensate for a global cold bias; we checked that it does not change our results. The concentration of greenhouse gases, the solar forcing, and aerosols are kept constant at present-day values. A period of $100 \mathrm{yr}$ is analyzed in each coupled simulation; the global radiative balance is close to zero in all simulations at the beginning of the analysis period. The coupled simulations will have the prefix CM4.

Finally, in four cases an additional coupled simulation is performed in which the atmospheric concentration of $\mathrm{CO}_{2}$ increases by $1 \% \mathrm{yr}^{-1}$, everything else being kept constant. The " $1 \% \mathrm{CO}_{2}$ " simulations start on 1 January of the corresponding coupled simulation and last for $80 \mathrm{yr}$. The last $40 \mathrm{yr}$ are analyzed; the $\mathrm{CO}_{2}$ concentration over that period has been multiplied by an average of 1.8.

To compare the GCM simulations with observations, we use the National Centers for Environmental Prediction-National Center for Atmospheric Research (NCEP-NCAR) reanalyses dataset (Kalnay et al. 1996), from 1979 through 2010. Using the longer-but less reliable-record from 1958 does not change the results. The global climate produced by these simulations is studied in Hourdin et al. (2012).

\section{Methods}

\section{a. Figure plotting conventions}

To help distinguish between the different types of simulations and resolutions, the following conventions are adopted for figures that display the results from several simulations at once:

- The colors give the resolution in latitude-71 points (black), 95 points (blue), and 144 points (red). The NCEP reanalysis is always in green.

- For a given resolution in latitude, simulations with the same number of points in longitude-for example, $96 \times 95-$ are in continuous lines or closed symbols.
The ones with more points in longitude-for example, $144 \times 95$ - are in dashed lines or open symbols.

- For scatterplots the simulations with imposed observed SSTs are denoted by squares, the coupled ones by circles, and the $1 \% \mathrm{CO}_{2}$ by triangles.

\section{b. Daily jet position}

Fluctuations of the Southern Hemisphere jet are measured using the daily jet latitude. The jet latitude is computed using the zonal wind at $850 \mathrm{hPa}$, a level that is representative of the eddy-driven jet and is not too much influenced by the physical parameterizations in the boundary layer. The wind is first zonally averaged, thereby providing a filter for the eddies, and then interpolated on a common $12^{\circ}$ latitude grid. The latitude of the zonal-mean jet is then taken as the center of the latitude band in which the wind speed is faster than the maximum speed minus $1 \mathrm{~m} \mathrm{~s}^{-1}$. This method removes possible local maxima located off the center of the broader jet, but the results are similar if using the raw latitude of the maximum wind speed.

As an example of the statistics obtained on the daily jet latitude, the year-round probability density functions (PDFs) for the imposed-SST simulations are shown on Fig. 1a, together with that from the NCEP reanalysis. One-degree-wide boxes were used to smooth the PDF and reduce noise. The peaks of the distributions move poleward with increasing resolution, especially in latitude (change of color, from black to blue to red). Figure $1 \mathrm{~b}$ shows for each simulation the variance of the distribution versus the jet latitude (defined as the mean of the distribution). There is a steady increase of the variance when the jet moves poleward, getting closer to the observed value. Coupled simulations lie on the same line as the ones with imposed SSTs, but with a jet shifted equatorward for a given resolution. As shown later, however, this annual-mean figure masks different behaviors in the winter and summer seasons.

\section{c. Southern annular mode}

The SAM is used to represent the dominant Southern Hemisphere midlatitude variability when the use of the zonal-mean jet latitude is less appropriate or when information on the zonal structure is needed. It is defined here as the first EOF of the monthly $850-\mathrm{hPa}$ zonal wind in the Southern Hemisphere, after weighting by the square root of the cosine of latitude but without prior zonal averaging. By convention, the positive phase of the SAM corresponds to negative pressure anomalies over the South Pole, or a poleward-shifted jet.

A daily SAM index can be constructed by projecting onto the SAM structure the daily $850-\mathrm{hPa}$ wind 

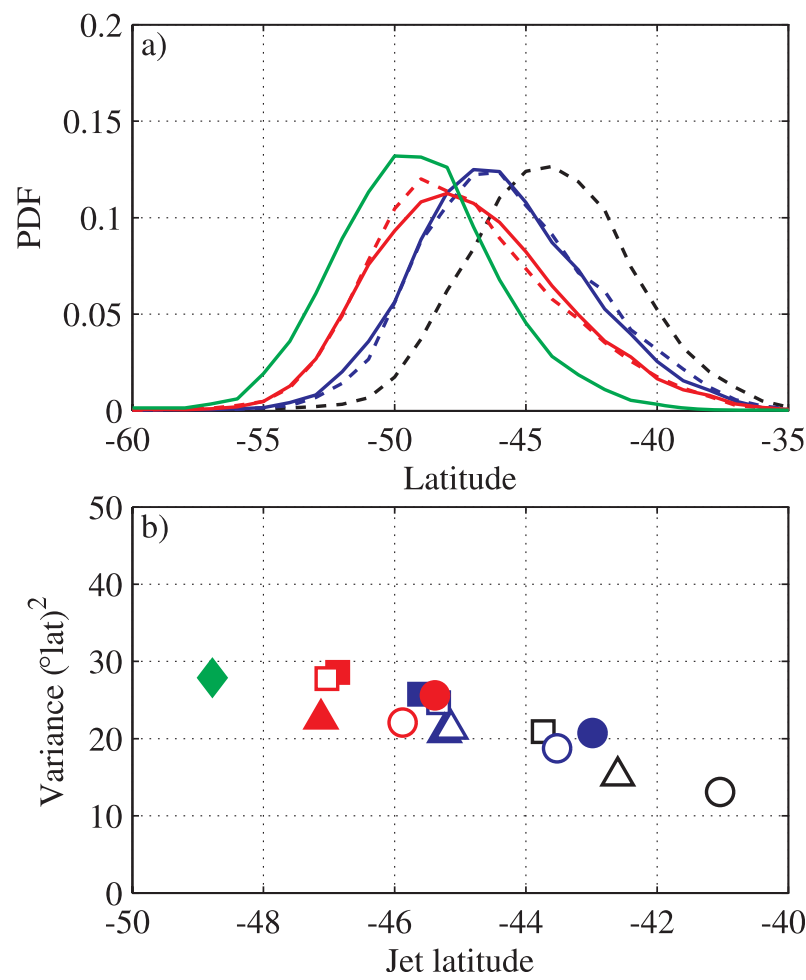

FIG. 1. (a) Distribution of the daily latitude of the jet for the whole year, in the imposed-SST simulations and the NCEP data. (b) Scatterplot for all the simulations and the NCEP data, of the variance of the jet latitude distribution vs the mean jet latitude (mean of the distribution). Plotting conventions are as follows: NCEP data are in green. Other colors show the resolution in latitude: 71 points (black), 95 points (blue), and 142 points (red). Simulations with the same number of points in latitude and longitude are continuous lines in (a) or solid symbols in (b); simulations with more points in longitude are dashed lines in (a) or open symbols in (b). Coupled simulations have circle symbols (triangles for $1 \% \mathrm{CO}_{2}$ ), while imposed-SST simulations have square ones.

anomalies from the seasonal cycle. A daily index of the eddy forcing of the SAM is also computed, by first computing the meridional convergence of eddy momentum flux (MFC), as

$$
\mathrm{MFC}=-\frac{1}{a \cos ^{2} \varphi} \partial_{\varphi}\left(u^{*} v^{*} \cos ^{2} \varphi\right),
$$

where $\varphi$ is the latitude, $a$ is the radius of the Earth, and the star denotes the wind departure from its zonal mean. The momentum flux convergence at the 200-hPa level (the level of its climatological maximum) is then projected onto the structure of the SAM $850-\mathrm{hPa}$ zonal wind anomalies to obtain the eddy-forcing index.

\section{Summer season}

The summer [December-February (DJF)] season is the period when the Southern Hemisphere midlatitude flow is closest to zonal symmetry, for the mean circulation as well as the storm tracks. Moreover, in the absence of a strong Hadley cell, the jet is close to being purely eddy driven. The climatological summer zonal winds at the 850- and 200-hPa levels are shown in Fig. 2a: there are only small variations in longitude, and the two jets lie on top of each other. The Southern Hemisphere summer season is therefore a good test bed for theories of the variability of eddy-driven jets.

The distribution of the daily jet latitude for the summer months is shown in Fig. 3 for the imposed-SST simulations. The distribution is shifting poleward for each increase in latitudinal resolution (change of color), reaching the observed latitude for the highest (142 points) resolution. When the resolution in longitude is increased instead, there is very little change in the latitude or the shape of the distribution. This behavior holds for all the simulations, as seen in Fig. 4, which show the mean summer jet latitude as a function of the resolution in latitude. The jet steadily moves poleward with increasing resolution for each type of simulation, with a more equatorward location for a given resolution in the coupled simulations (squares) than with imposed SSTs (circles). The $1 \% \mathrm{CO}_{2}$ (triangles) are at intermediate positions, shifted poleward compared to the corresponding coupled simulation. The position of the jet in the complete set of simulations spans $8^{\circ}$ of latitude.

The reasons for this behavior were explored by Guemas and Codron (2011) using an idealized Held and Suarez (1994) setup with the LMDZ4 dynamical core. They found that the latitude shift could be attributed to a general increase in wave activity, with larger eddy momentum fluxes pushing the jet poleward, a behavior previously observed by Held and Phillipps (1993) at lower resolutions. With an increase of the resolution in longitude, the increase in wave activity was accompanied by an increased tendency for poleward propagation of the waves, which prevented a jet shift. With the full GCM, the jet also tends to move slightly poleward when increasing the resolution in longitude, especially in coupled simulations. This change in the model behavior may be linked to a warming of the tropics in the full GCM, possibly caused by the physical parameterizations (Hourdin et al. 2012).

According to Fig. 4, the simulations closest to observations are the high-resolution, imposed-SST ones with 142 points in latitude (red squares), which have almost the same zonal-mean jet distribution as NCEP. A map of the associated summer-mean zonal wind is shown in Fig. $2 \mathrm{~b}$ : it is indeed very close to the observed one. For comparison, the winds from the CM4-96 $\times 71$ simulation (open black circle in Fig. 4) are shown in Fig. 2c. They are clearly different, with a jet that is too narrow 
a) NCEP

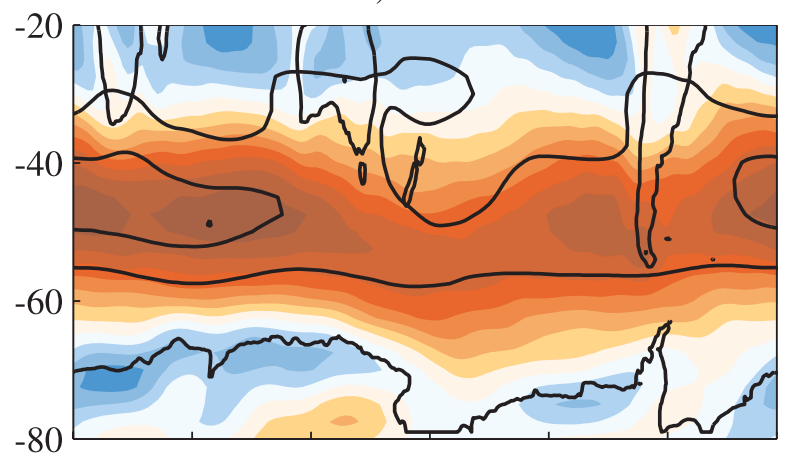

b) LMDZ4-144×142

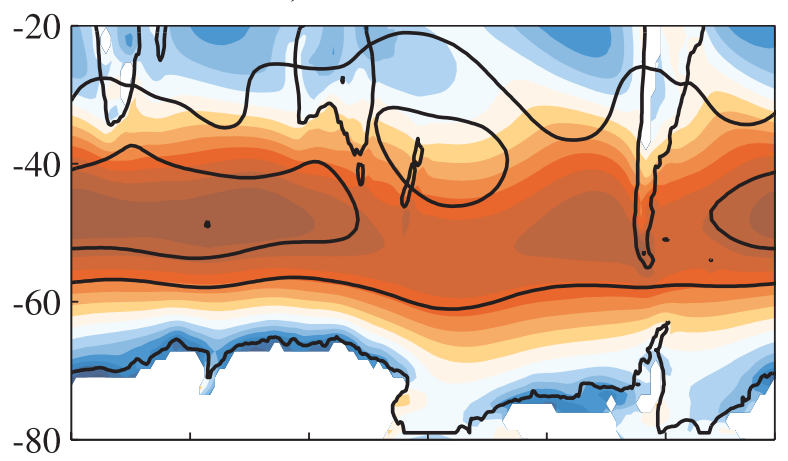

c) $\mathrm{CM} 4-96 \times 72$

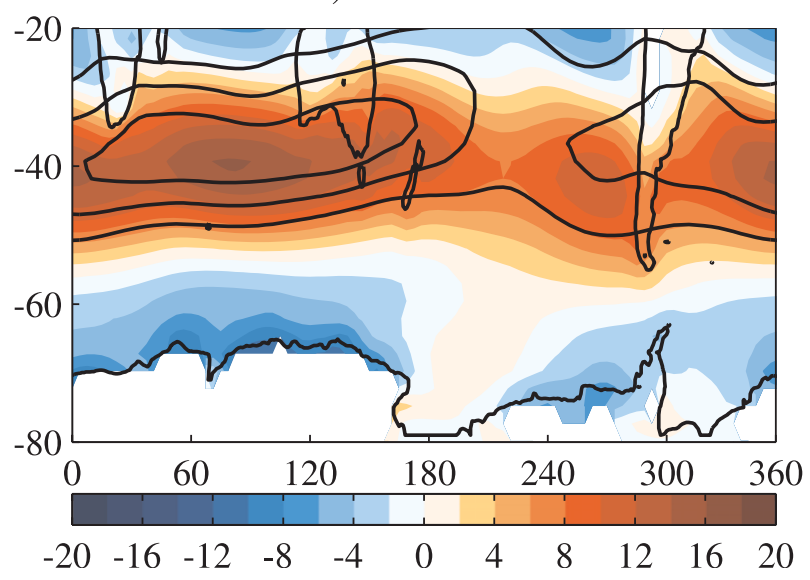

FIG. 2. Mean summer (DJF) zonal wind over the Southern Ocean, at $850 \mathrm{hPa}$ (color) and $200 \mathrm{hPa}$ (contours at 22, 32, and $42 \mathrm{~m} \mathrm{~s}^{-1}$ ), for (a) NCEP, and simulations (b) LMDZ4-144 × 142 and (c) CM4-96 × 72 .

and too strong at $200 \mathrm{hPa}$, and located at a lower latitude.

\section{a. Jet variability}

More statistics of the jet variability in the different simulations are shown in Fig. 5. The horizontal axis is in all cases the mean latitude of the jet. The daily jet latitude is used as a basic index, but a daily SAM index

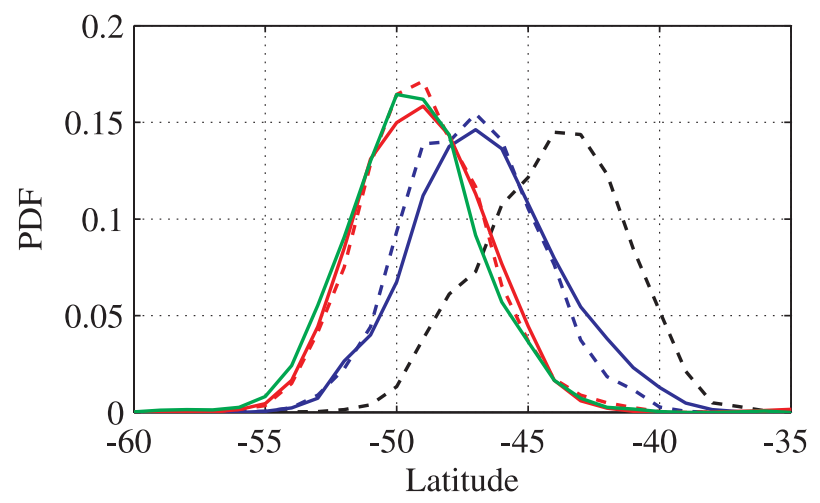

FIG. 3. Distribution of the daily jet latitude for imposed-SST simulations and NCEP data (green) in summer (DJF).

would yield the same results: in all simulations as in observations, the summer SAM represents, to first order, a shift of the jet around its mean position (not shown).

The variance of the distribution (Fig. 5a) shows only little spread around the observed value with no systematic tendency (the vertical scale is the same for variance plots in summer, winter, and whole year). The skewness (Fig. 5b) is negative for the most equatorward jets (i.e., skewed toward poleward positions) and then increases toward the observed value of zero when the jet moves poleward. This could suggest the existence of a barrier against jet movements deep into the subtropics. The mean speed of the jet at $850 \mathrm{hPa}$, taken each day at its actual latitude, is shown in Fig. 5c. It increases slightly when the jet moves poleward, and it is overestimated by $0.5 \mathrm{~m} \mathrm{~s}^{-1}$ at the highest resolutions.

The decorrelation time scale of jet movements is shown in Fig. 5e. It was computed by fitting the lagged autocorrelation function of the daily jet latitude with an exponential over the first 10 days. The time scale decreases with the jet latitude, converging toward the observed value as observed by Kidston and Gerber (2010) and Barnes and Hartmann (2010b). Note that the range of latitudes covered here is the same as for all the CMIP3 models they used.

Only two simulations display a systematically lower time scale than expected given their jet latitude: they are the coupled simulations with a larger number of points in longitude than in latitude (open circles). The reason for this behavior is unclear, but a distinct feature of these simulations is a very strong cold bias in the midlatitude SSTs compared to the tropical ones, which is reduced when the number of points in latitude is larger (Hourdin et al. 2012). Perhaps the strong subtropical SST gradient has an anchoring effect that could prevent long excursions of the jet (Sampe et al. 2010). 


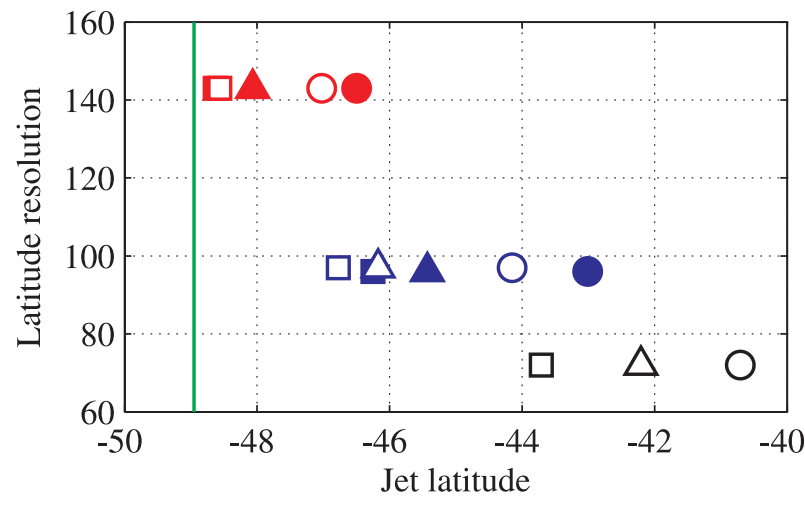

FIG. 4. Scatterplot for all the simulations of the mean summer jet latitude vs the resolution in latitude. Circles are coupled simulations; squares are imposed-SST simulations. Green line marks the latitude of the observed (NCEP) jet.

The monthly variance explained by the SAM (Fig. 5f) behaves exactly as the time scale of jet movements, decreasing from up to $40 \%$ to the observed $15 \%$. This is expected if the time scale of other modes of variability does not change with resolution: the mode with the longer time scale will then become more prominent at lower frequencies. The results in Fig. 5f suggest this effect dominates the changes of explained variance.

Despite a factor of 2 spread of the time scale or explained monthly variance, the variance of the daily jet latitude changes very little between the simulations. This apparent discrepancy can be resolved by looking at the variance of the eddy forcing (Fig. 5d): the variance of $m$ increases with the jet latitude, perhaps reflecting the increase in wave activity observed by Guemas and Codron (2011). Again, using the notations from Lorenz and Hartmann (2001), the frequency spectrum $Z Z^{*}$ of the SAM index $z$ can be written as

$$
Z Z^{*}=\frac{\tilde{M} \tilde{M}^{*}}{\omega^{2}+\left(\tau^{-1}-b\right)^{2}},
$$

where $\tilde{M} \tilde{M} *$ is the variance spectrum of the random component $\tilde{m}$ of $m$ (which is supposed to be close to white), $\omega$ is the frequency, and $b$ is the eddy feedback coefficient. The SAM or jet latitude variance can thus be increased by either increasing $b$, which will act at lower frequencies [periods longer than the decorrelation time scale, $\left.\omega \ll\left(\tau^{-1}-b\right)\right]$ or else the random eddy forcing $\tilde{M}$, which will act equally at all frequencies. For monthly values, the changes of the eddy feedback dominate; however, for the total variance, a compensation seems to be occurring as the jet moves poleward in the different simulations, with decreasing feedback (and time scale) but increasing random forcing. This compensation also occurs in the two coupled simulations with short time scales (open circles): they both have a larger total eddy forcing, and a similar variance. It is not clear whether this compensation is an intrinsic property of the flow. The jet movements could be limited to a range of latitudes by external factors, such as the width of the baroclinic zone, but the jet position varies with resolution, and its range of movements is not constant in other seasons.

\section{b. Response to $\mathrm{CO}_{2}$ increase}

The four $1 \% \mathrm{CO}_{2}$ simulations show a systematic poleward shift of the summer jet compared to the control coupled simulation at the same resolution (Fig. 4). The amplitude of this shift was found by Kidston and Gerber (2010) and Barnes et al. (2010) to be correlated in CMIP3 models to both the initial latitude of the mean jet and the time scale of the annular mode. These two quantities are themselves correlated in CMIP3 models, with equatorward jets having more persistent fluctuations and a stronger response to $\mathrm{CO}_{2}$. In our coupled simulations, however, the time scale alternatively increases and decreases when the jet moves poleward (Fig. 5e). It is thus possible to estimate which is the best predictive variable for the response to increased $\mathrm{CO}_{2}$.

This jet shift between the control and $1 \% \mathrm{CO}_{2}$ simulations is shown in Fig. 6 as a function of either the initial jet latitude or the time scale of jet fluctuations in the control simulation. No systematic relation appears: in each case, three out of four simulations reproduce the annual-mean behavior observed in CMIP3 models of larger response for low latitude or long time scale, with a different outlier. We tried using the latitude of the mean jet instead of the mean latitude, or the explained variance instead of the decorrelation time scale. The fit can be improved to some extent with the "right" pick, but the basic conclusion remains. The largest change comes from using the SAM time scales from the simulations with increased $\mathrm{CO}_{2}$, which behave very differently from the control ones (Fig. 5e); the results are then similar to using the jet latitude.

Even with versions of the same model differing only in horizontal resolution, the relationships between jet latitude, variability time scale, and amplitude of the response to $\mathrm{CO}_{2}$ forcing are thus not straightforward. A possible explanation lies in the "forcing" of the annular mode [term $F$ in Eq. (3)]. A different projection of the response to $\mathrm{CO}_{2}$ increase onto the annular mode structure could yield different amplitudes of the response (Ring and Plumb 2008) at different resolutions. The mean temperature changes between the $\mathrm{CO}_{2}$ and control simulations are almost independent of resolution (Hourdin et al. 2012). The spatial correspondence 

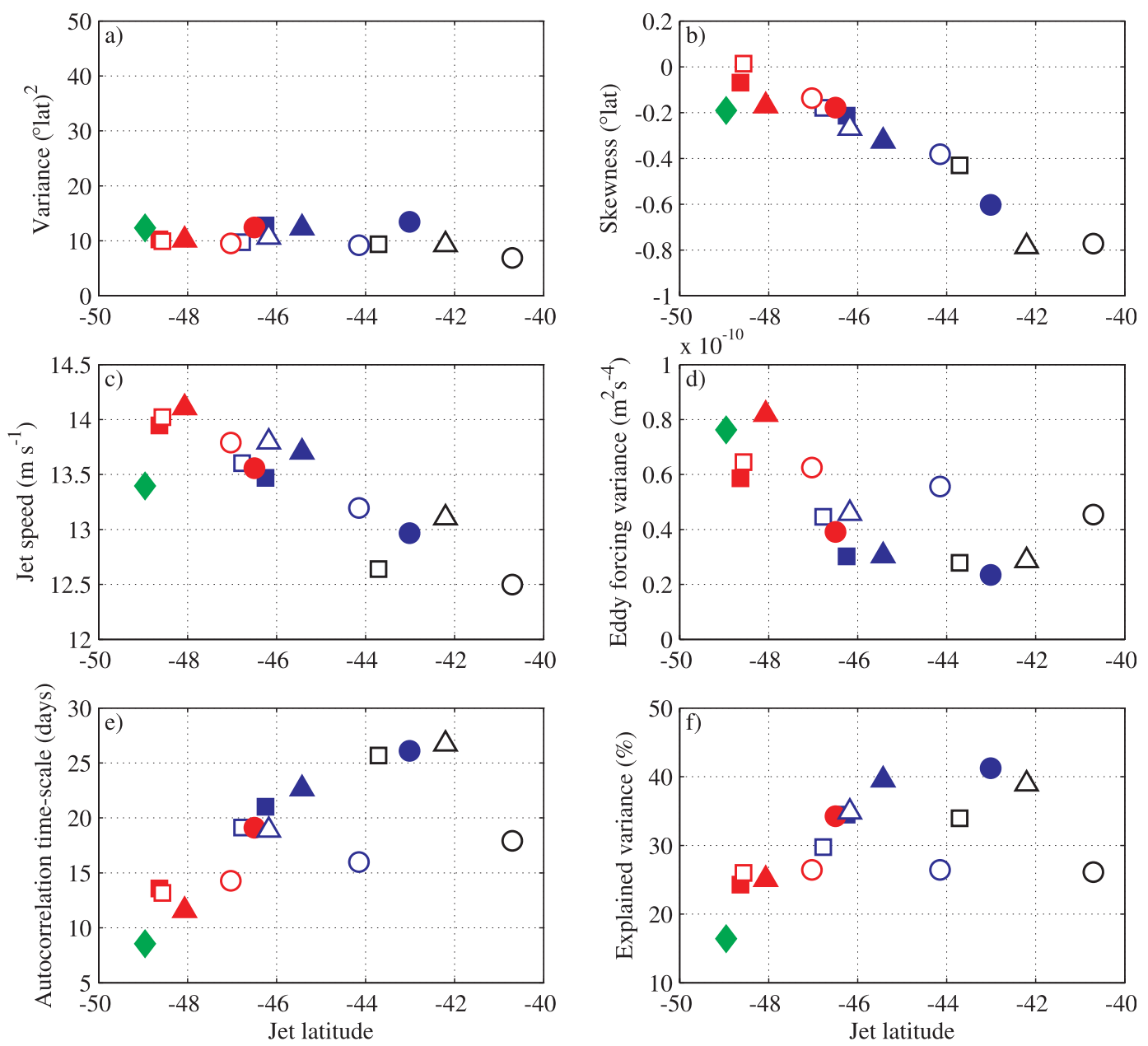

FIG. 5. Scatterplots of different characteristics of the summer jet and its variability, plotted for each simulation vs the mean latitude of the jet: (a) variance and (b) skewness of the distribution, (c) mean speed of the jet, (d) variance of $m$ (see text), (e) autocorrelation time scale of the daily fluctuations in the jet latitude, and (f) variance explained by the first EOF of the 850-hPa monthly-mean zonal wind (the SAM). NCEP is shown as a green diamond; otherwise, for each type of symbol, the resolution uniformly increases from black to blue to red. Detailed symbol meanings are given in section $3 \mathrm{a}$ and in the legend of Fig. 1.

between the warming pattern and the jet position could also have an influence, but it is difficult to measure directly.

\section{Winter season}

In winter [June-August (JJA)], the distribution of the latitude of the zonal-mean jet evolves in a different way with resolution (Fig. 7a): the poleward movement of the distribution mean is much less pronounced than in summer and stops well short of the observed one, but the variance of the jet position increases rapidly instead or being quasi stationary (Fig. 7b). It seems that the variance would reach the observed one if the simulated jet position were extrapolated. The corresponding plot for the whole year (Fig. 1) is thus misleading as it averages two opposite behaviors: large shifts with near-constant variance in summer and small shifts with increasing variance in winter.

Taking the zonal mean may, however, mask important features of the winter jet variability, as departures of the mean state from zonal symmetry are larger in this season and the mean jet is not everywhere at the same latitude. Figure $8 \mathrm{a}$ shows the mean winter $850-\mathrm{hPa}$ and $200-\mathrm{hPa}$ zonal winds over the Southern Ocean. Compared to the summertime circulation, a strong subtropical jet appeared at the upper level, centered over the Indian and western Pacific Oceans. The surface jet is strongest over the Atlantic and Indian Oceans at $45^{\circ}-50^{\circ} \mathrm{S}$, where it lies under the upper-level jet. It then becomes progressively 

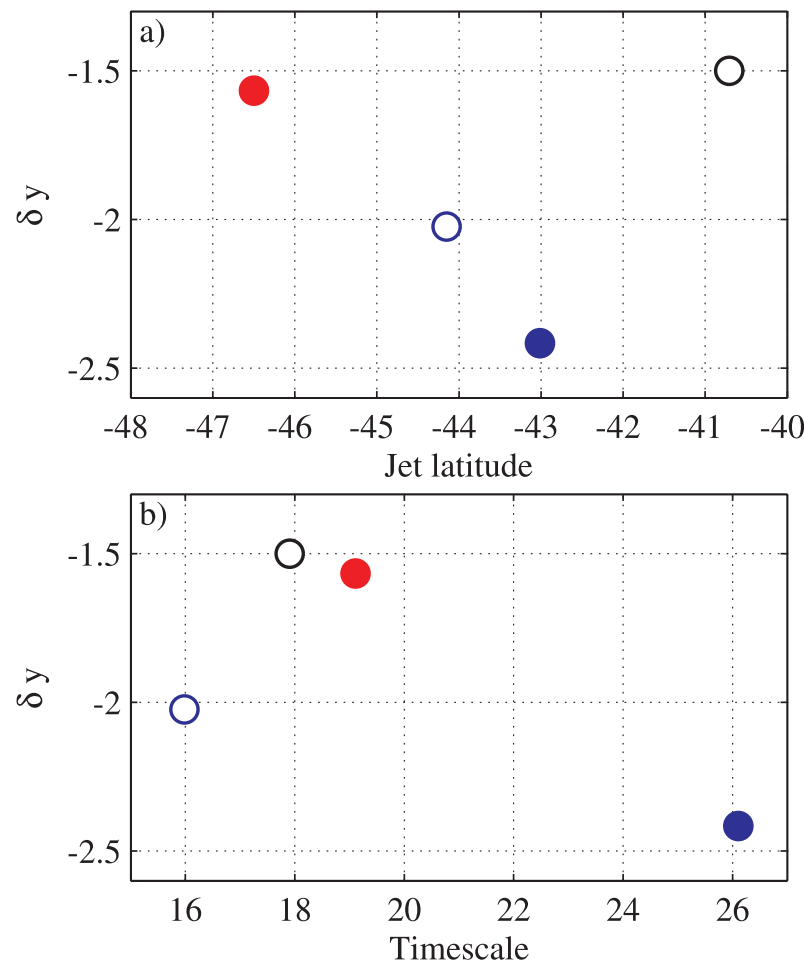

FIG. 6. Differences $\delta y$ in the mean jet latitude between $1 \% \mathrm{CO}_{2}$ and control simulations, as a function of (a) the latitude of the jet and (b) the time scale of jet fluctuations in the corresponding control simulation.

weaker and moves south to $60^{\circ} \mathrm{S}$ in the Pacific. At the same longitudes, a secondary maximum appears to the north, located on the poleward flank of the subtropical jet.

As in summer, the wind simulated by two extreme simulations is shown in Figs. 8b,c. Unlike in summer, despite some improvement with the higher resolution, strong biases remain. Both simulations have a too-strong subtropical jet and a too-weak midlatitude jet. Close to the surface, the jet is located too far equatorward in the Indian Ocean and extends too far eastward, while in the Pacific the two extrema are well located but the wind speed is too strong at the equatorward maximum and too weak at the poleward one.

To separate the different behaviors of the jet at different longitudes, we now focus on the two sectors delineated by dashed lines in Fig. 8: the Indian Ocean sector, characterized by a single strong eddy-driven jet; and the Pacific sector, where the jet splits in two. The mean jet latitude in the Indian sector is shown in Fig. 9a for all the simulations. As in summer, there is a general poleward shift tendency, but here no simulation reaches the observed position. The jet in simulations with increased $\mathrm{CO}_{2}$ is also always displaced poleward. In the
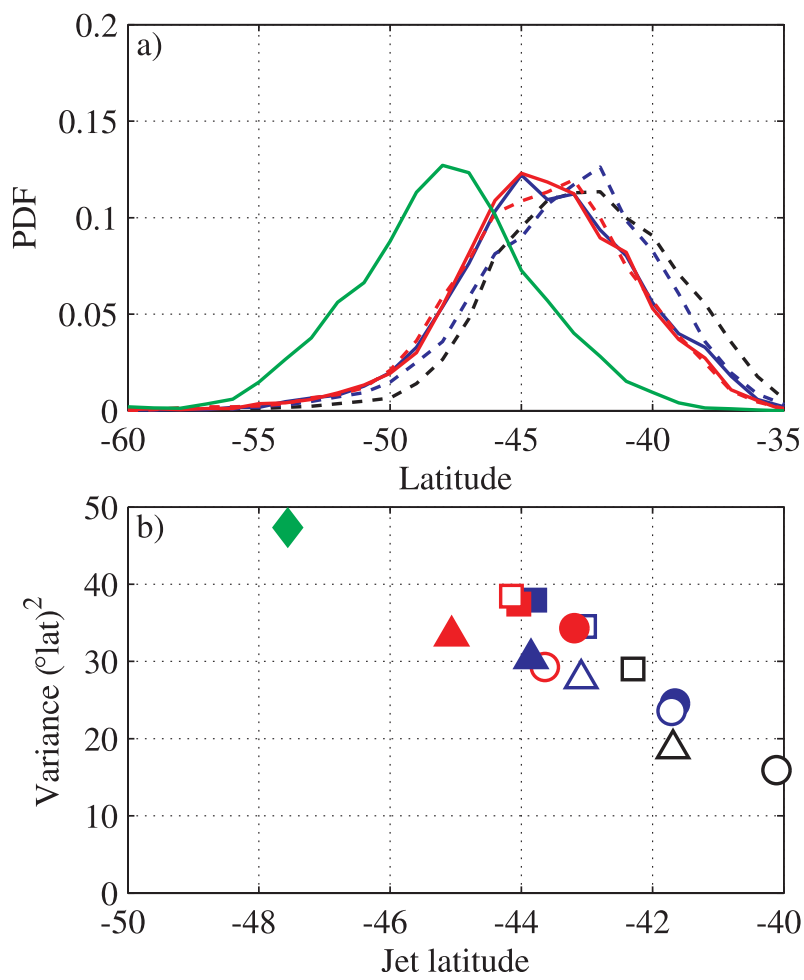

FIG. 7. (a) Distribution of the daily latitude of the jet for the winter months, in the imposed-SST simulations and the NCEP data. (b) Scatterplot for all of the simulations and the NCEP data, of the variance of the jet latitude distribution vs the mean jet latitude (mean of the distribution).

Pacific sector, the positions of the two jets are stationary in different simulations, but their speed changes with resolution. We thus show (Fig. 9b) the differences between the mean wind speeds at $35^{\circ}$ and $60^{\circ} \mathrm{S}$, which are the peak positions of the subtropical and midlatitude jets. As for the Indian sector, there is some improvement with resolution, but the subtropical jet remains too strong - and the midlatitude one too weak-compared to observations even at the highest resolution.

\section{a. Jet variability}

We now check the influence of these mean-state biases on the simulation of the dominant variability, represented here by the SAM to capture the zonal structure. Zonal-mean composites of the 850-hPa zonal wind over each sector for the NCEP data are shown in Figs. 10a,b, for the winter-mean state as well as for the positive and negative phases of the SAM (months in which the SAM index is larger than one standard deviation are used). Over the Indian Ocean sector, the SAM is a latitude shift of the jet, with a slight strengthening in the poleward position. Over the Pacific sector, there is no change in the positions of the jet extrema at $35^{\circ}$ and $60^{\circ} \mathrm{S}$ but an 
a) NCEP

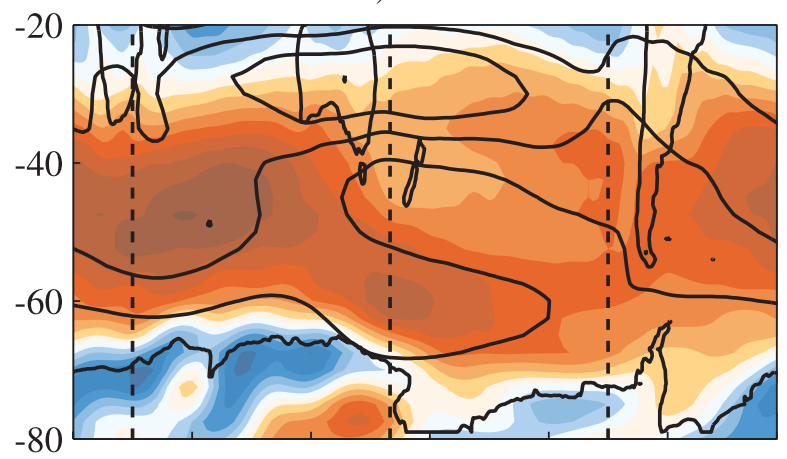

b) LMDZ4-144×142

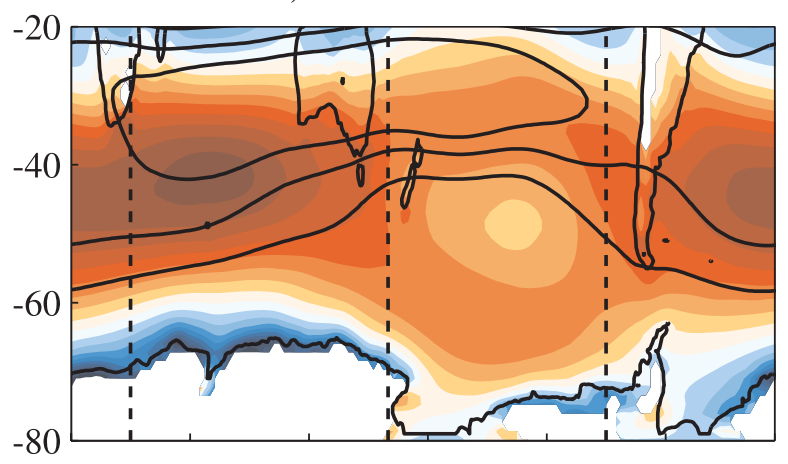

c) $\mathrm{CM} 4-96 \times 72$

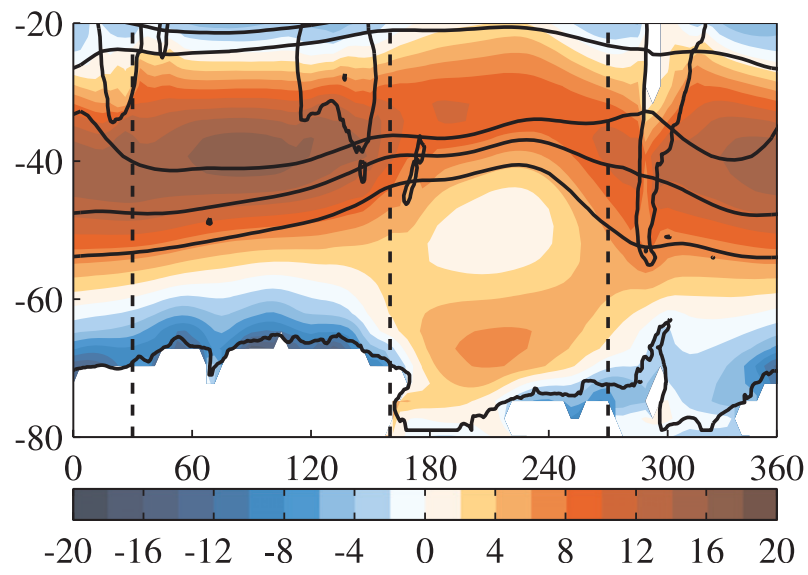

FIG. 8. Mean winter zonal wind over the Southern Ocean, at the 850-hPa level (color) and 200-hPa level (contours at 22, 32, and $42 \mathrm{~m} \mathrm{~s}^{-1}$ ) for (a) NCEP, and simulations (b) LMDZ4-144 × 142 and (c) CM4-96 × 72 .

opposite change in amplitude, with the poleward jet being much stronger in the positive phase and the subtropical one being slightly stronger in the negative phase. This seesaw behavior of the SAM in the Pacific was observed by Codron (2007), who also showed that it was well correlated with jet shifts over the Indian Ocean sector.

The next panels of Fig. 10 show the same composites for the LMDZ4-144 × 142 and CM4-96 × 71
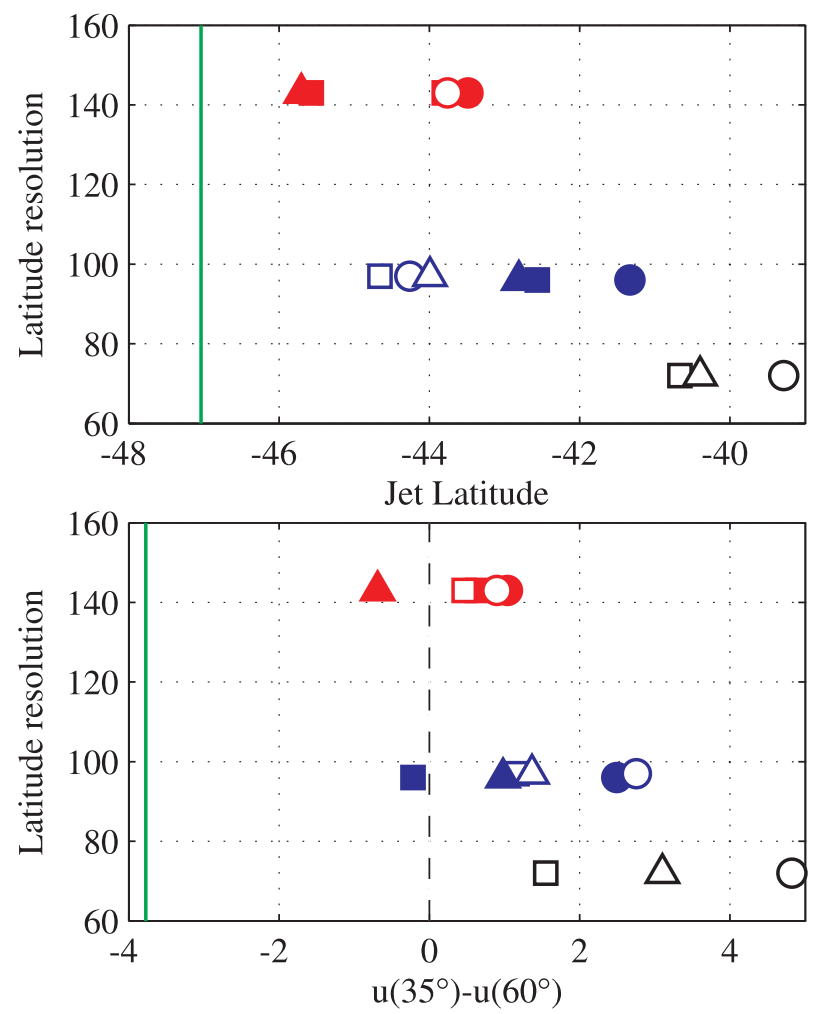

FIG. 9. Scatterplot for all of the simulations in winter of the resolution in latitude vs (top) the mean jet latitude in the Indian Ocean sector, and (bottom) the difference between the wind speed at $35^{\circ}$ and $60^{\circ} \mathrm{S}$ in the Pacific sector. Circles are coupled simulations; squares are imposed-SST simulations. Green line marks the observed (NCEP) value.

simulations. For the first one (Figs. 10c,d), the SAM composites look very similar to the observed ones: in the Indian Ocean the SAM is a pure jet shift, but around a latitude that is located equatorward of the observed one. In the Pacific, the SAM is a seesaw between the two observed locations, but with a relative amplitude that is always too strong at the equatorward position. While the mean state of the LMDZ4-144 $\times 142$ simulation is very close to a negative phase of the observed SAM, the simulated variability around that mean state thus resembles the observed one. In the second simulation (Figs. 10e,f), the mean biases are even stronger, with a jet equatorward of $40^{\circ} \mathrm{S}$ in the Indian Ocean and an extremely weak and poorly located poleward maximum in the Pacific. As a result, while the SAM is still a jet shift in the Indian Ocean, it becomes more a fluctuation of the subtropical jet over the Pacific: the observed relation between dominant variability and mean state here is partially lost.

\section{b. Response to $\mathrm{CO}_{2}$ increase}

The coupled simulations with increased $\mathrm{CO}_{2}$ display a systematic poleward jet shift over the Indian Ocean 

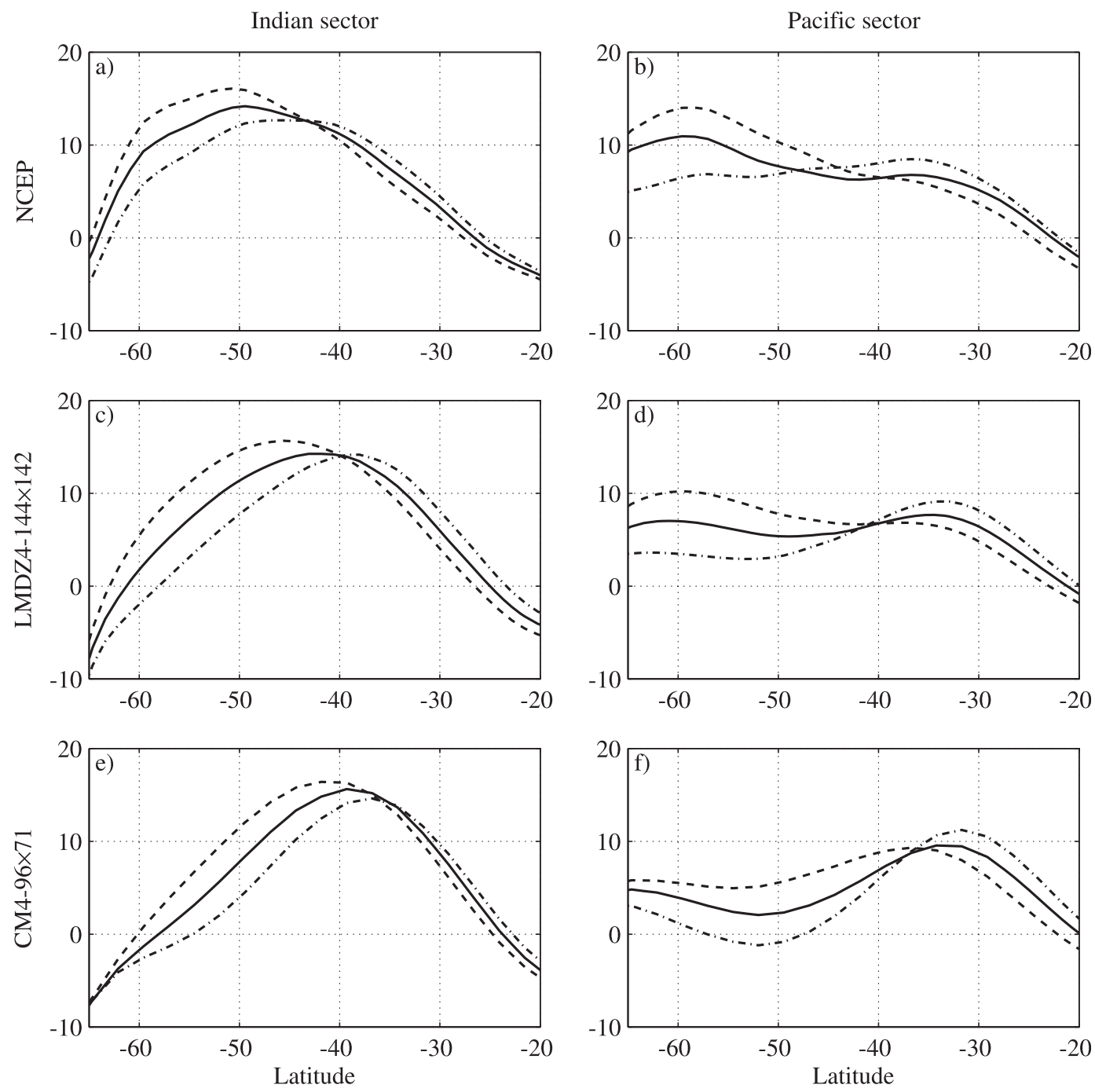

FIG. 10. Zonal averages of the 850-hPa zonal wind over the (a),(c),(e) Indian Ocean and (b),(d),(f) Pacific sectors for winter: mean wind (solid), and composites of the positive (dashed) and negative (dot-dashed) SAM phases. (a),(b) NCEP, (c),(d) LMDZ4-144 × 142 simulation, (e),(f) CM4-96 × 71 simulation.

sector, and a relative strengthening of the midlatitude jet over the Pacific sector, as can be seen in Fig. 9. From the results of the previous section, this behavior yields a tendency toward the positive phase of the SAM in both sectors. To judge how well the response to the $\mathrm{CO}_{2}$ increase projects onto the SAM structure, Fig. 11 compares the regression on the $850-\mathrm{hPa}$ wind on the SAM index to the difference between the $1 \% \mathrm{CO}_{2}$ and control simulations. The $96 \times 96$ resolution is shown but others behave similarly. The response to the $\mathrm{CO}_{2}$ increase is shifted slightly poleward-perhaps because it is compared to the control SAM structure-but the two are otherwise very similar; the surface wind response to the $\mathrm{CO}_{2}$ increase thus projects very strongly onto the SAM, including the zonal asymmetries.

\section{Conclusions}

A consistent set of experiments was completed with the LMDZ general circulation model at varying horizontal resolutions, both with imposed SSTs at the surface and coupled with an oceanic GCM. Additional experiments with increased $\mathrm{CO}_{2}$ were also performed in the latter case. The range of simulated positions of the surface winds over the Southern Ocean is of the same order as the one covered by the CMIP3 models, and larger than either the response to the $\mathrm{CO}_{2}$ increase or to intraseasonal variability.

The response of the midlatitude circulation to increasing resolution was studied for the two extreme summer (DJF) and winter (JJA) seasons. In summer, the 

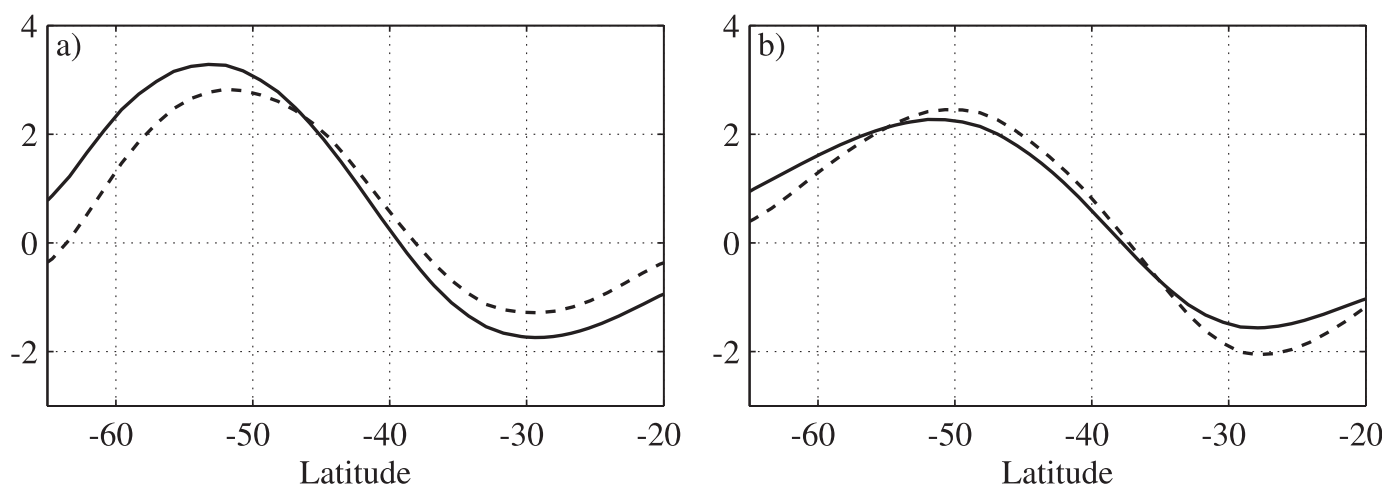

FIG. 11. Zonal wind at the 850-hPa level in winter: regression on the SAM index (solid), and difference between the $1 \% \mathrm{CO}_{2}$ and control simulations (dashed). Zonal averages over the (a) Indian Ocean and (b) Pacific sectors. The resolution used is $96 \times 96$ points.

mean circulation is almost zonally uniform and the lowfrequency variability is dominated by latitude shifts of the zonal-mean jet. In winter, the zonal asymmetries are stronger, and the dominant mode of variability (the SAM, defined by the first EOF) has a more complex relation with the mean state: it changes from a latitude shift of the jet to a seesaw between two preferred positions in the Pacific.

The main conclusions for both seasons are summarized below.

- The changes of the simulated circulation as the resolution increases project strongly onto the SAM. In summer, the jet moves poleward, especially with increased resolution in latitude, and reaches the observed position with the highest resolution. In winter, the simulated winds also improve, but the changes-poleward shift in the Indian Ocean sector and stronger midlatitude jet in the Pacific-remain too small to bring the model in line with observations.

- The dominant variability in each simulation displays the same relationship with the mean state as observed: jet shifts in summer and a Pacific seesaw in winter. This remains true even for mean-state biases larger than the typical internal variability, except in one case when the Pacific midlatitude winter jet completely disappears.

- The detailed statistics of jet fluctuations in summer display in most simulations a simple dependence on the mean jet latitude. They improve steadily with resolution and converge to the observed ones for the best simulations. The dynamics underlying the variability, as measured by the autocorrelation time scale or eddy forcing, behave similarly with a progressive rebalancing of the eddy feedback and random forcing components when the mean jet moves poleward.

- The response to the transient $\mathrm{CO}_{2}$ increase also projects strongly on the SAM structure, including in the winter in the Pacific sector. No systematic relationship between the amplitude of the response and the control mean state or variability could be obtained; however, there is a tendency toward larger jet shifts in summer with an equatorward jet or more persistent annular mode. The statistical significance with only four simulations is, however, weak.

In summer, it thus seems that doubling the original resolution in latitude is enough to get a very realistic simulation of the Southern Hemisphere jet, including details such as the time scale or higher moments of the jet fluctuations. It could be that in summer, both the mean jet position and its variability are determined by eddy-mean flow interactions, so that resolving the dominant baroclinic waves is both necessary and sufficient.

In winter, however, the same increase in resolution does not bring as much benefit. Eddy-mean flow interactions are probably not as dominant in that season, and the influence of the tropics is much stronger; indeed, the Pacific sector, where the subtropical jet is strongest, arguably shows the least improvement. Biases in tropical heating, for example, may have a strong influence on the midlatitudes; however, they are more dependent on the model physics and thus will not necessarily improve with horizontal resolution.

Acknowledgments. We thank the two anonymous reviewers for their constructive remarks, which helped improve the paper. We would also like to thank MarieAlice Foujols, who performed all the simulations used in this paper; and Virginie Gumas, who helped in the preliminary analysis. The simulations were run at the Institut du Développement et des Ressources en Informatique Scientifique (IDRIS). 


\section{REFERENCES}

Barnes, E. A., and D. L. Hartmann, 2010a: Dynamical feedbacks of the southern annular mode in winter and summer. J. Atmos. Sci., 67, 2320-2330.

$\longrightarrow$, and - 2010b: Testing a theory for the effect of latitude on the persistence of eddy-driven jets using CMIP3 simulations. Geophys. Res. Lett., 37, L15801, doi:10.1029/2010GL044144.

$\longrightarrow,-$ D. M. W. Frierson, and J. Kidston, 2010: Effect of latitude on the persistence of eddy-driven jets. Geophys. Res. Lett., 37, L11804, doi:10.1029/2010GL043199.

Chen, G., and R. A. Plumb, 2009: Quantifying the eddy feedback and the persistence of the zonal index in an idealized atmospheric model. J. Atmos. Sci., 66, 3707-3720.

Codron, F., 2007: Relations between annular modes and the mean state: Southern Hemisphere winter. J. Atmos. Sci., 64, 3328-3339.

Gerber, E. P., and G. K. Vallis, 2007: Eddy-zonal flow interactions and the persistence of the zonal index. J. Atmos. Sci., 64, 32963311.

Gillett, N. P., and D. W. J. Thompson, 2003: Simulation of recent Southern Hemisphere climate change. Science, 302, 273 -275, doi:10.1126/science.1087440.

Guemas, V., and F. Codron, 2011: Differing impacts of resolution changes in latitude and longitude on the midlatitudes in the LMDZ atmospheric GCM. J. Climate, 24, 5831-5849.

Held, I. M., and P. J. Phillipps, 1993: Sensitivity of the eddy momentum flux to meridional resolution in atmospheric GCMs. J. Climate, 6, 499-507.

_- and M. J. Suarez, 1994: A proposal for the intercomparison of the dynamical cores of atmospheric general circulation models. Bull. Amer. Meteor. Soc., 75, 1825-1830.

Hourdin, F., and Coauthors, 2006: The LMDZ4 general circulation model: Climate performance and sensitivity to parametrized physics with emphasis on tropical convection. Climate Dyn., 27, 787-813, doi:10.1007/s00382-006-0158-0.

— grid configuration on the climate and sensitivity of the IPSL-CM5A coupled model. Climate Dyn., doi:10.1007/ s00382-012-1411-3, in press.

Hurrell, J. W., J. J. Hack, D. Shea, J. M. Caron, and J. Rosinski, 2008: A new sea surface temperature and sea ice boundary dataset for the Community Atmosphere Model. J. Climate, 21, $5145-5153$.
Kalnay, E., and Coauthors, 1996: The NCEP/NCAR 40-Year Reanalysis Project. Bull. Amer. Meteor. Soc., 77, 437-471.

Kidston, J., and E. P. Gerber, 2010: Intermodel variability of the poleward shift of the austral jet stream in the CMIP3 integrations linked to biases in 20th century climatology. Geophys. Res. Lett., 37, L09708, doi:10.1029/2010GL042873.

Krinner, G., and Coauthors, 2005: A dynamic global vegetation model for studies of the coupled atmosphere-biosphere system. Global Biogeochem. Cycles, 19, GB1015, doi:10.1029/ 2003 GB002199.

L'Heureux, M. L., and D. W. J. Thompson, 2006: Observed relationships between the El Niño-Southern Oscillation and the extratropical zonal-mean circulation. J. Climate, 19, 276287.

Lorenz, D. J., and D. L. Hartmann, 2001: Eddy-zonal flow feedback in the Southern Hemisphere. J. Atmos. Sci., 58, 33123327.

Marti, O., and Coauthors, 2010: Key features of the IPSL ocean atmosphere model and its sensitivity to atmospheric resolution. Climate Dyn., 34, 1-26, doi:10.1007/s00382-009-0640-6.

Perlwitz, J., S. Pawson, R. L. Fogt, J. E. Nielsen, and W. D. Neff, 2008: Impact of stratospheric ozone hole recovery on Antarctic climate. Geophys. Res. Lett., 35, L08714, doi:10.1029/ 2008GL033317.

Ring, M. J., and R. A. Plumb, 2008: The response of a simplified GCM to axisymmetric forcings: Applicability of the fluctuationdissipation theorem. J. Atmos. Sci., 65, 3880-3898.

Robinson, W. A., 2006: On the self-maintenance of midlatitude jets. J. Atmos. Sci., 63, 2109-2122.

Sampe, T., H. Nakamura, A. Goto, and W. Ohfuchi, 2010: Significance of a midlatitude SST frontal zone in the formation of a storm track and an eddy-driven westerly jet. J. Climate, 23, 1793-1814.

Sen Gupta, A., and M. H. England, 2006: Coupled oceanatmosphere-ice response to variations in the southern annular mode. J. Climate, 19, 4457-4486.

Son, S.-W., and Coauthors, 2010: Impact of stratospheric ozone on Southern Hemisphere circulation change: A multimodel assessment. J. Geophys. Res., 115, D00M07, doi:10.1029/ 2010JD014271.

Watterson, I. G., 2007: Southern annular modes simulated by a climate model: Patterns, mechanisms, and uses. J. Atmos. Sci., 64, 3113-3131. 